Bio -grafía Escritos sobre la Biología y su Enseñanza.

Edición Extra-Ordinaria.

Memorias del 1 Congreso Nacional de Investigación en Enseñanza de la Biología.

VI Encuentro Nacional de Investigación en Enseñanza de la Biologia y la

Educación Ambiental. ISSN 2027 1034. P. p. 333-341.

\title{
Didáctica de la biotecnología en la escuela
}

\section{Biotechnology Didactics in the school}

POR: Robinson Roa Acosta ${ }^{1}$

\section{Resumen}

En este escrito se presentan algunos aspectos relacionados con la didáctica de la biotecnología, ante el hecho de su crecimiento acelerado y sus múltiples aplicaciones en el sector agropecuario, salud, industria y ambiente, entre otros. Se pone de manifiesto la polisemia, estatus epistemológico y la posibilidad de entender la biotecnología como un objeto de enseñanza en la escuela.

Con el fin de hacer visibles estas características, se estructura y propone un taller para profesores de ciencias naturales en ejercicio y futuros profesores de la misma área, que les permita reflexionar y problematizar sus concepciones sobre la biotecnología y su enseñanza escolar.

Palabras clave: enseñanza de la biotecnología, biotecnología, conocimiento del profesor, estatus epistemológico.

\section{Abstract}

In this paper we present some aspects related to Biotechnology didactics to the fact its quick growth and its many applications in agriculture, health, industry and environment, among others. It shows polysemy, epistemological status and the possibility of understanding biotechnology as a subject to be taught in school.

In order to make visible these characteristics, a workshop for science teachers and future teachers practicing in the same area, allowing them to reflect and problematize their conceptions on biotechnology and school teaching was structured and proposed.

Key words: biotechnology technology, biotechnology, teacher's knowledge, epistemological status.

\footnotetext{
${ }^{1}$ Estudiante doctorado interinstitucional en educación. Universidad Pedagógica Nacional. Universidad Francisco José de Caldas. Universidad del Valle. Línea de Investigación Conocimiento Profesional de Profesor de Ciencias. robinsonroa@hotmail.com. Este taller se construye como parte de los desarrollos alcanzados de la tesis doctoral.
} 
Bio -grafía Escritos sobre la Biología y su Enseñanza.

Edición Extra-Ordinaria.

Memorias del 1 Congreso Nacional de Investigación en Enseñanza de la Biología.

VI Encuentro Nacional de Investigación en Enseñanza de la Biologia y la

Educación Ambiental. ISSN 2027 1034. P. p. 333-341.

\section{Introducción}

La biotecnología cada vez con mayor intensidad influye en la vida de las personas en aspectos tan importantes tales como la salud y alimentación, en tanto la política y la economía se encuentran sujetas a las investigaciones adelantas por diversos grupos de investigación en el mundo. Sin embargo, la enseñanza en los colegios y en la formación de profesores de ciencias naturales todavía es muy exigua; en los casos en los cuales es enseñada no se tiene referentes epistemológicos fundamentados sobre los cuales hacer de la misma un objeto de enseñanza; las concepciones sobre lo que representa para la sociedad y ambiente, la vida y lo vivo, política y economía, enseñanza y aprendizaje, se encuentran por hacerse explicitas como conocimiento que funde una de las razones de investigación de la didáctica de las ciencias.

Es decir, la educación en ciencias naturales -particularmente la biología- se encuentra atrasada en cuanto a observar la biotecnología como objeto de enseñanza, al mismo tiempo que en las investigaciones didácticas. En tal sentido, el taller pretende indagar, reflexionar y analizar con los participantes sobre sus concepciones acerca de la didáctica y la enseñanza de la biotecnología.

Para dicho análisis se abordan elementos teóricos y de reflexión, organizados a partir de la estructura sintáctica y sustantiva (Schwab (1978) citado en Shulman, 1986): asuntos relacionados con las definiciones sobre la biotecnología, las posibilidades de encontrar su estatus epistemológico como disciplina, ciencia, interdisciplina o multidisciplina. En lo que atañe a la enseñanza de la biotecnología la discusión se centra en los contenidos, estrategias y finalidades.

\section{Variedad de definiciones sobre la biotecnología}

Ante los adelantos científicos desarrollados por la biotecnología, y las múltiples implicaciones políticas y económicas que han desencadenado diversidad de aplicaciones en la industria farmacéutica, medicina y producción agrícola, entre otras, autores como Aycardi (1986); Sasson (1989); Abrams (1993); Strohman (1997), reconocen que el conocimiento biotecnológico, resultado de las investigaciones, ha impactado desde mediados del siglo XX. En concordancia, Rifkin (1998) y Fukuyama (2003) agregan que se espera para el siglo XXI un desarrollo y crecimiento mucho mayor, no sólo en la naturaleza de su investigación sino también, particularmente, en sus aplicaciones a la producción agrícola, salud, industria alimentaria y sostenimiento de los ecosistemas. 
Memorias del 1 Congreso Nacional de Investigación en Enseñanza de la Biología.

VI Encuentro Nacional de Investigación en Enseñanza de la Biología y la Educación Ambiental. ISSN 2027 1034. P. p. 333-341.

Así también, en la medida en que el conocimiento se vuelve más especifico, las fronteras entre las disciplinas de vuelven menos visibles, al igual que los conceptos y definiciones son más perplejos y complejos. Esto tiene sus raíces en las circunstancias en las cuales el conocimiento se moviliza y dinamiza a un ritmo de tiempo que no deja mucho espacio para que los filósofos, epistemólogos y profesores, elaboren sus propias construcciones y las puedan compartir con las comunidades de investigadores. De tal manera, el número de publicaciones son aceleradas y las definiciones diversificadas respecto a un mismo "aparentemente" conocimiento. La biotecnología es un buen ejemplo.

En cuanto a la polisemia que presenta, Lemkow (2000) comenta que algunos estudios de opinión pública revelan un conjunto más bien amplio de actitudes hacia la biotecnología, que es como un concepto paraguas bajo el cual se agrupan técnicas y aplicaciones que cubren diversos campos. Resalta, que muchas reuniones científicas empiezan con largos debates a propósito de su definición más adecuada. Esto se ejemplifica por la coexistencia de 41 definiciones diferentes en documentos de la Unión Europea. Así pues, si este era el número de definiciones hasta el año 2000, es posible advertir que este número definiciones en la actualidad haya aumentado, por cuanto las aplicaciones se han hecho evidentes para muchos campos más. Es decir, se podría decir que se encuentran tantas definiciones como intereses industriales, comerciales, económicos, políticos, legales y de formación académica en los diferentes niveles de educación.

Según Budd (1989) historiador de la biotecnología (citado por Lemkow (2000), el término fue usado por primera vez en 1919, por el ingeniero agrónomo húngaro Karl Ereki quien la definió como: "Todas la líneas de trabajo con las cuales los productos son elaborados a partir de la materia prima con la utilización de organismos vivos." (p. 35).

\section{Estatus epistemológico de la biotecnología}

Es evidente que existen dificultades para definir la biotecnología en los ámbitos científico, cotidiano y escolar. Adicionalmente, se pueden encontrar casos en los que para referirse a la misma se ha acuñando términos como: disciplina, disciplina horizontal, disciplina multidisciplinar o interdisciplinar. 
Bio -grafía Escritos sobre la Biología y su Enseñanza.

Edición Extra-Ordinaria.

Memorias del 1 Congreso Nacional de Investigación en Enseñanza de la Biología.

VI Encuentro Nacional de Investigación en Enseñanza de la Biología y la

Educación Ambiental. ISSN 2027 1034. P. p. 333-341.

Se pueden citar algunos ejemplos tales como:

Puigdomènech (2000), quien la declara como una disciplina horizontal, porque necesita de un conjunto de conexiones y técnicas de procedencia muy diversa, porque se aplica a campos también de gran diversidad.

Bolaños, et al (2003), afirman que "a lo largo de la historia la biotecnología ha desarrollado, en sí misma, metodologías de trabajo de carácter científico, empleando principalmente microorganismos o células vegetales y animales de aplicación industrial para resolver problemas que afectan al hombre; por su impacto e implicaciones, esta disciplina multidisciplinar se está constituyendo en un elemento cultural del siglo XXI." (p. 189).

Roa y Urbina (2005), destacan que "para la International Union of Pure and Applied Chemestry (IUPAC) la biotecnología involucra las aplicaciones de bioquímica, biología, microbiología e ingeniería química. Este carácter de trabajo interdisciplinario y sus resultados, revierten vertiginosamente en el mundo del conocimiento, y al unísono también, con un gran impacto a nivel sociocultural, económico y político."

Para Roa, et al (2008), "la historia y la filosofía de la Biología muestran como a través de sus distintos episodios ha desarrollado y aportado conceptos que, en reunión con otros de diferente naturaleza epistemológica -Física y Química, por lo menos-, conducen al surgimiento de la biotecnología como campo interdisciplinario... en la biotecnología, la compilación disciplinar sinérgica que se desarrolla promueve la intersección de múltiples conocimientos que se sustentan sin la cual perdería las características esenciales que la hacen ser como es-, la convierte en un campo de conocimiento complejo..." (p. 79).

Así también, existen autores que clasifican la biotecnología como una disciplina. Es el caso de Aznar (2000) quien señala que "Debido a los rápidos avances de la ciencia, hablamos ahora de un nuevo término que se ha ido implantando en el ámbito de la educación en ciencias: biotecnología, en el que no sólo se incluyen los conceptos tradicionalmente ligados a la genética. Esta nueva disciplina aporta un alto grado de contextualización a toda la terminología científica y a sus aplicaciones, lo que debería contribuir a un mayor conocimiento sobre la materia, a una mayor significatividad de los aprendizajes y a los procesos de formación de actitudes." (p. 12). 
Bio -grafía Escritos sobre la Biología y su Enseñanza.

Edición Extra-Ordinaria.

Memorias del 1 Congreso Nacional de Investigación en Enseñanza de la Biología.

VI Encuentro Nacional de Investigación en Enseñanza de la Biología y la

Educación Ambiental. ISSN 2027 1034. P. p. 333-341.

Lo que se puede observar como elemento común en los autores citados (Aznar, 2000; Puigdomènech, 2000; Bolaños, et al. 2003; Roa y Urbina, 2005; Roa, et al. 2008) es la participación de diferentes disciplinas en la constitución de la biotecnología. Sin embargo, tal y como se ha señalado antes, parece no existir un acuerdo en la comunidad académica respecto a su propio estatus epistemológico.

Roa y Valbuena (2009) ya habían advertían que se evidenciaba que la biotecnología se estaba planteando de manera diversa: como ciencia, disciplina, multidisciplina, entre otras, o simplemente no se le asigna un estatus definido. Señalan también que si se compara con la Biología, Química, Física o Matemática, es un campo de conocimiento relativamente joven; podría decirse que inicia su reconocimiento con el modelo de la estructura del ADN, aunque sus prácticas tienen su origen desde varios siglos atrás.

Por su parte, Grevechova, et al (1995) ponen de relieve que la biotecnología demanda del conocimiento de diferentes ciencias y disciplinas (Biología, Bioquímica, Fisiología, Microbiología, Ingeniería Genética, Enzimología, Biología Molecular, etc.).

En ese mismo sentido Castellanos, et al (1996), plantean que "Con el transcurrir del tiempo se fueron haciendo populares los conceptos como el de microbiología industrial, tecnología bioquímica, biología aplicada, etc. Para agrupar todas estas nacientes áreas del conocimiento y de la producción hace algo más de dos décadas atrás se empezó a introducir el término de biotecnología. Probablemente la necesidad de hacer más evidente la importancia de esta naciente ciencia fue motivada por los descubrimientos en áreas como la enzimología, la biología molecular y la genética. ( $p$ 51)... fueron los profesionales de otras áreas los que tomaron la iniciativa de su desarrollo. Hoy en día la divulgación de la biotecnología como una ciencia con su propia identidad y su propia comunidad académica y profesional, enriquecida con elementos de muchas disciplinas complementarias, es evidente en los países desarrollados y es una necesidad en los países de la región. ( $\mathrm{p} 52$ ). ... a principios de los ochenta [en Colombia] se empezaba a hablar tímidamente de la biotecnología como ciencia independiente, en otros países ya se contaba con adelantos significativos e investigaciones en curso." ( $p 53$ ).

En esta última cita se pone en evidencia una vez más que la biotecnología está constituida de manera ecléctica por conocimientos de diversa procedencia, no obstante, parece ser muy apresurado señalar que es una ciencia con su propia identidad y a renglón seguido escribir que es enriquecida con elementos de muchas disciplinas. Precisamente es paradójico que se diga que tiene identidad a 
Bio -grafía Escritos sobre la Biología y su Enseñanza.

Edición Extra-Ordinaria.

Memorias del 1 Congreso Nacional de Investigación en Enseñanza de la Biología.

VI Encuentro Nacional de Investigación en Enseñanza de la Biología y la

Educación Ambiental. ISSN 2027 1034. P. p. 333-341.

la vez que se complementa con varias disciplinas, lo cual podría llevar a pensar lo contrario.

Roa y Valbuena (2009) en relación con la participación de diversas ciencias y disciplinas científicas en la biotecnología, plantean diferentes niveles para entenderlas: como su simple concurrencia, como resultado de su interacción, como el enriquecimiento a partir de su participación, como un espacio interdisciplinar. En ese sentido, habría que hacer distinción entre la biotecnología como ciencia, disciplina, etc. y la biotecnología como producto de la ciencia, disciplina, etc.

Se identifica así la participación de varias disciplinas que la convierten en un conocimiento diverso y disperso en teoría, no obstante, que en las prácticas científicas están presentes implícitamente. Se puede decir que hay ambigüedad sobre la identidad de la biotecnología y sobre las construcciones epistemológica y filosófica que conduzcan a la constitución de su objeto de investigación. Hasta el momento no se encuentran argumentos sólidos que presenten los fundamentos epistemológicos y filosóficos al respecto. De hecho es pertinente preguntarse, de ser posible entender la biotecnología como disciplina, ¿cuál sería su objeto de investigación? Para de esta poder dilucidar cuál podría ser el objeto de enseñanza.

En ese sentido, es prioritario rastrear las características relativas a sus estructuras sintáctica (entendida como los criterios que se emplean para la validación del conocimiento) y sustantiva (que constituye los principios básicos que caracterizan la disciplina y la diferencian de otras, es decir, esto es identificar los elementos propios de su estructura interna), que permitan el análisis de su objeto (s) de enseñanza.

\section{Establecimiento del objeto de enseñanza de la biotecnología}

Abordar el objeto de enseñanza de la biotecnología, en la práctica, es por ahora un obstáculo, primero porque las investigaciones en didáctica se encuentran en estado embrionario, y segundo -aunque no es consecuencia de lo anteriorporque aún no se han formalizado la constitución del estatus epistemológico y las acercamiento filosóficos son escasos. Sólo se ha escrito de manera muy informal que es una disciplina, multidisciplina, ciencia o interdisciplina, no se ha entrado a la construcción de las estructuras sintáctica y sustantiva que permitan poder dar cuenta de la naturaleza de dicho conocimiento. 
Bio -grafía Escritos sobre la Biología y su Enseñanza.

Edición Extra-Ordinaria.

Memorias del 1 Congreso Nacional de Investigación en Enseñanza de la Biología.

VI Encuentro Nacional de Investigación en Enseñanza de la Biología y la

Educación Ambiental. ISSN 2027 1034. P. p. 333-341.

Estos aspectos afectan profundamente las posibilidades de abordar los contenidos y elaborar los currículos, en tanto no se puede clarificar si la biotecnología tiene su origen en la tecnología, biología, física o química o es resultado de diferentes objetos de investigación, lo cual igualmente dificulta reconocer el objeto de enseñanza. Por lo menos para el caso de la tecnología y la biología ya se pueden encontrar discusiones respecto a cuál le corresponde incluir la biotecnología como contenido de enseñanza.

García y Roa (2009) señalan que los aspectos históricos y paradigmáticos de la biotecnología la tornan llamativa para el público en general y los estudiantes, por lo que es un campo de conocimiento sobre el cual la enseñanza de la Biología debe poner la lupa y observar cuidadosamente su alcance, no solo en lo social sino también en su didáctica: proponiendo innovación y actualización a los profesores para su enseñanza, aprendizaje, evaluación.

Así pues, se puede decir que desde la década de los años noventa hasta el momento las investigaciones y propuestas, al respecto de su enseñanza, se han hecho esperar, sin embargo, se encuentran en estado embrionario, de manera fragmentada y difusa, por lo que es necesario establecer los aspectos que, desde la didáctica de las ciencias, se ha investigado o propuesto, para así vislumbrar sus posibilidades (grupos de investigación, propuestas, innovaciones) y connotaciones económicas, bioéticas, políticas, ambientales, etc., fundamentadas en la historia, epistemológicas y filosofía. De esto mismo surgirían las posibilidades de identificar perspectivas, problemas y tipos de investigación, percepción y conocimiento público, potencialidad didáctica.

\section{Metodología taller}

El taller se desarrolla para un grupo de máximo quince profesoras (es) ejercicio y estudiantes a profesores del área de ciencia naturales. Se encuentra dividido en básicamente tres momentos:

- Introducción: se presentan los objetivos del taller y los referentes teóricos que lo orientan.

- Actividad individual: los participantes contestan una pregunta que posteriormente se socializa entre todos los participantes.

- Actividad grupal: en esta los participantes diseñan una actividad relacionada con la enseñanza de un tema en biotecnología y elaboran una cartelera que sintetice dicho diseño. Posteriormente se realiza una plenaria.

- Actividad de cierre: se establecerán las conclusiones y se hará entrega de un material de apoyo relacionado con el taller. 
Memorias del 1 Congreso Nacional de Investigación en Enseñanza de la Biología. VI Encuentro Nacional de Investigación en Enseñanza de la Biología y la Educación Ambiental. ISSN 2027 1034. P. p. 333-341.

Durante la socialización el tallerista está formulando preguntas que posibiliten la reflexión de los participantes respecto a la didáctica y la enseñanza de la biotecnología, haciendo uso de los recursos teóricos hasta el momento desarrollados.

\section{Agradecimientos}

Se agradece al Doctor Édgar Orlay Valbuena Ussa, coordinador de la línea de investigación Conocimiento Profesional del Profesores Ciencias, por los aportes realizados en la elaboración del taller.

\section{BIBLIOGRAFÍA}

- ABRAMS, P. (1993). Analyzing biotech's past, present, and future. Bio/Technology. Abril vol. 11, p. 450-451.

- AYCARDI, E. (1986). Alcance, desarrollo y perspectivas de la Biotecnología en el país. Colombia. Ciencia y Tecnología. Agosto-octubre, vol. 4 (4), p. 28-29.

- AZNAR, V. (2000). ¿Qué conocemos sobre la Biotecnología?. Alambique Didáctica de la Ciencias Experimentales. N 25, Julio, 9-14.

- BOLAÑO, P.; PULIDO, M.; GARCÍA, Y. ROA, R. (2003). Investigaciones escolares de Biotecnología en educación media, una forma de integrar ciencia, tecnología y sociedad. Revista Tecne, Episteme y Didaxis. Número extra, p. 187-188. Memorias. 2ํㅡㄹ Congreso sobre Formación de Profesores de Ciencias. Universidad Pedagógica Nacional, Bogotá.

- CASTEllaNOS, O.; SALCEDO, L., y GREVECHOVA, R. (1996). Importancia del factor educacional en el desarrollo sostenido de la Biotecnología. Revista Estudios en Pedagogía. Julio-septiembre 9 (2), p. 50-65.

- FUKUYAMA, F. (2003). El fin del hombre. Consecuencias de la revolución biotecnológica. Barcelona, España: Ediciones B.

- GARcía, Y y ROA, R; (2009). Desarrollo de investigaciones en la enseñanza de la Biotecnología. Una revisión. Memorias: I Congreso Nacional de Investigación en Educación en Ciencias y Tecnología. 
Memorias del 1 Congreso Nacional de Investigación en Enseñanza de la Biología. VI Encuentro Nacional de Investigación en Enseñanza de la Biología y la Educación Ambiental. ISSN 2027 1034. P. p. 333-341.

Asociación Colombiana para la Investigación en Ciencia y Tecnología, Bogotá.

pp. 1-6. http://educyt.wikispaces.com/file/view/Desarrollo+de+investigaciones+en

- GREVECHOVA, R.; SALCEDO, L., y CÁRDENAS, F. (1995). Biotecnología y educación básica. Actualidad Educativa, mayo-junio, 2 (7), p. 35-40.

- LEMKOW, L. (2000). La sociedad ante los riesgos biotecnológicos. Revista Sostenible?, (2) 31-43.

- PUIGDOMÈNECH, P. (2000). Què és la Biotecnología?. Revista Sostenible?. (2), 25-30.

- RIFKIN, J. (1998). The Biotech Century: Harnessing The Gene and Remaking the World. EE. UU.: Putmann/Tarcher, 288 p.

- ROA, R y VALBUENA, È. (2009). Hacia la estructuración de la educación en Biotecnología como problema didáctico de investigación. Primer Congreso Nacional de Investigación en Educación en Ciencias y Tecnología. Asociación Colombiana para la Investigación en Ciencia y Tecnología, Bogotá.

- ROA, R, GARCíA, Y., y CHAVARRO, C. (2008). Formación de profesores de Biología través de la Biotecnología. Revista Educación y Educadores. 11 (2), 69-88. Universidad de la Sabana.

- ROA, R., y URBINA, J. (2005). Reflexión en torno a la introducción de la Biotecnología en la educación media y básica. Memorias. Revista Tecne, Episteme y Didaxis. Número extra, p. 192-193. 20 Congreso sobre Formación de Profesores de Ciencias. Universidad Pedagógica Nacional, Bogotá.

- SASSON, A. (1989). Biotecnologías y los países en desarrollo: promesas y desafíos. Colombia. Ciencia y Tecnología, enero-marzo, 7 (1), p. 4-8.

- SHULMAN, L. (1986). Paradigms and research programs in the study of teaching: A contemporary perspective. In: WITTROCK, M. (Ed.) Handbook of Research on Teaching. New York: Macmillam. p. 3-36.

- STROHMAN, R. (1997). The coming Kuhnian revolution in biology. Nature Biotechology. 15, p. 194-200. 